\title{
Adaptive Neural-Sliding Mode Control of Active Suspension System for Camera Stabilization
}

\author{
Feng Zhao, Mingming Dong, Yechen Qin, Liang Gu, and Jifu Guan \\ School of Mechanical Engineering, Beijing Institute of Technology, Beijing 100081, China \\ Correspondence should be addressed to Liang Gu; guliang@bit.edu.cn
}

Received 10 March 2015; Revised 29 April 2015; Accepted 30 April 2015

Academic Editor: Marco Alfano

Copyright (c) 2015 Feng Zhao et al. This is an open access article distributed under the Creative Commons Attribution License, which permits unrestricted use, distribution, and reproduction in any medium, provided the original work is properly cited.

\begin{abstract}
The camera always suffers from image instability on the moving vehicle due to the unintentional vibrations caused by road roughness. This paper presents a novel adaptive neural network based on sliding mode control strategy to stabilize the image captured area of the camera. The purpose is to suppress vertical displacement of sprung mass with the application of active suspension system. Since the active suspension system has nonlinear and time varying characteristics, adaptive neural network (ANN) is proposed to make the controller robustness against systematic uncertainties, which release the model-based requirement of the sliding model control, and the weighting matrix is adjusted online according to Lyapunov function. The control system consists of two loops. The outer loop is a position controller designed with sliding mode strategy, while the PID controller in the inner loop is to track the desired force. The closed loop stability and asymptotic convergence performance can be guaranteed on the basis of the Lyapunov stability theory. Finally, the simulation results show that the employed controller effectively suppresses the vibration of the camera and enhances the stabilization of the entire camera, where different excitations are considered to validate the system performance.
\end{abstract}

\section{Introduction}

Camera stabilization is very important for visual guidance in autonomous vehicle, and the goal of image stabilization is to remove unwanted motion from dynamic camera sequences [1]. The better image-acquisition process will increase the feasibility and reliability of the process and analysis afterward. Current efforts in the research of image stabilization techniques can be broadly classified as three major categories: optical stabilization [2], digital image stabilization [3], and mechanical stabilization [4]. The ability of vibration amplitude control in optical and digital image stabilization is relatively limited. Different from former two stabilizers, mechanical stabilization involves stabilizing the entire camera, not just the image. It is suitable for large camera moving situation. In the condition of the camera fixed on the top of the car, the vertical vibration amplitude of the camera needs to be reduced. Therefore, active suspension system (ASS) is a kind of mechanical stabilization that can suppress the large vertical vibration caused by road roughness.

Active suspension system has been widely used in both military and civil vehicles [5-7]. Compared with the conventional suspension, the force actuator is added to provide energy to the suspension system, which enables the suspension to control the attitude of the vehicle body [8]. In previous works such as $[9,10]$, they ignored the actuator dynamics. However, the nonlinear dynamic of the hydraulic actuator should be considered to improve the performance of the control system [11]. The control strategy of active suspension system has attracted many researchers' attention in the past few decades. Different control approaches have been applied, such as Ho [12], LQG control [13], fuzzy control [14], and artificial neural networks [15]. The road disturbances, the measurement errors, and the dynamic uncertain model are the key factors that affect the control performance.

In reality, the camera's vibrations are inevitable and unpredictable. The vehicle body can provide a stable platform for the camera to detection of the obstacles with application of active suspension system. For a nonlinear active suspension system with bounded uncertainty and external disturbance, the sliding mode control (SMC) has the advantage of robustness to improve the antidisturbance ability of the system. In [16], the state predictors along with SMC technique are applied to active suspension system, which consider the time delay of the data transmission. Mixed control strategies are 
proposed in [17], and an enhanced adaptive self-fuzzy sliding mode controller for a quarter-car active suspension system is presented. Furthermore, an adaptive sliding controller is used with the function approximation approach for nonlinear system containing bounded unknown time-varying uncertainties in [18]. Besides the SMC strategy, neural network approach has received increasing attention mainly due to its advantages of nonlinear mapping properties and its ability to deal with uncertainty [19]. However, in traditional multilayer neural controller, the relatively complex structure of neuron network takes much time for computation and weight adjustment. An adaptive neural network with learning and self-tuning ability could be used to overcome the drawbacks.

This paper lays an emphasis on designing a controller with a quarter-car active suspension to suppress vertical vibration of the camera so that the displacement of the sprung mass changes as small as possible. In this way, we propose a sliding mode controller combined with adaptive neural networks approach to stabilize the image captured area of the camera. In the meantime, the dynamics of the hydraulic actuator is considered and can track the desired force with PID controller in the inner loop. Owing to the parameters of the model which are nonlinear, conventional adaptive schemes are not applicable. Adaptive radial basis function neural network (RBFNN) [20,21] is employed to make the controller robustness against system uncertainties and the update laws of the weighting matrix are derived on the basis of the Lyapunov stability theory. The closed loop stability and asymptotic convergence performance can be guaranteed. Finally, the effectiveness of the proposed control approach is validated by simulations under different road profiles.

This paper is organized as follows. Section 2 gives suspension system dynamic. Section 3 develops the adaptive NN controller. In Section 4, the results of computer simulation of the controller are presented. Section 5 concludes this paper.

\section{Suspension System Dynamic and Problem Formulation}

This paper considers the quarter-car model [22] with a camera installed on the top of vehicle as shown in Figure 1. The camera is rigidly connected with the vehicle body. The sprung mass $m_{b}$ represents the car body and unsprung mass is $m_{w}$. The tire is modeled as a linear spring with stiffness $k_{t} . c_{p}$ and $k_{s}$ are the linear damping and stiffness of the suspension system, respectively. Variables $x_{b}, x_{w}$, and $x_{r}$ are the displacement of the body, wheel, and road profile, respectively. A hydraulic actuator between the sprung and unsprung masses can exert a force $F_{a}$.

The dynamic equations of the suspension system can be expressed as

$$
\begin{aligned}
& m_{b} \ddot{x}_{b}+k_{s}\left(x_{b}-x_{w}\right)+c_{p}\left(\dot{x}_{b}-\dot{x}_{w}\right)-F_{a}=0, \\
& m_{w} \ddot{x}_{w}+k_{t}\left(x_{w}-x_{r}\right)+k_{s}\left(x_{w}-x_{b}\right)+c_{p}\left(\dot{x}_{w}-\dot{x}_{b}\right) \\
& \quad+F_{a}=0 .
\end{aligned}
$$

The electrohydraulic actuator comprises a servo-valve and a hydraulic cylinder as shown in Figure 2. Detailed

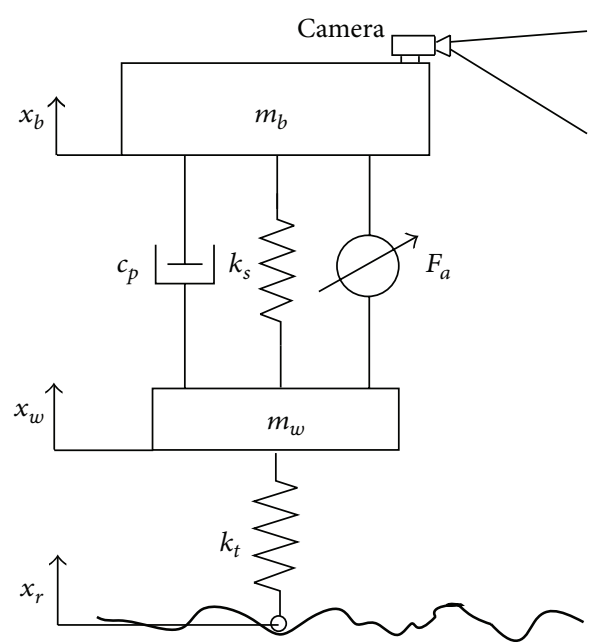

FIGURE 1: Quarter-car model with an active suspension.

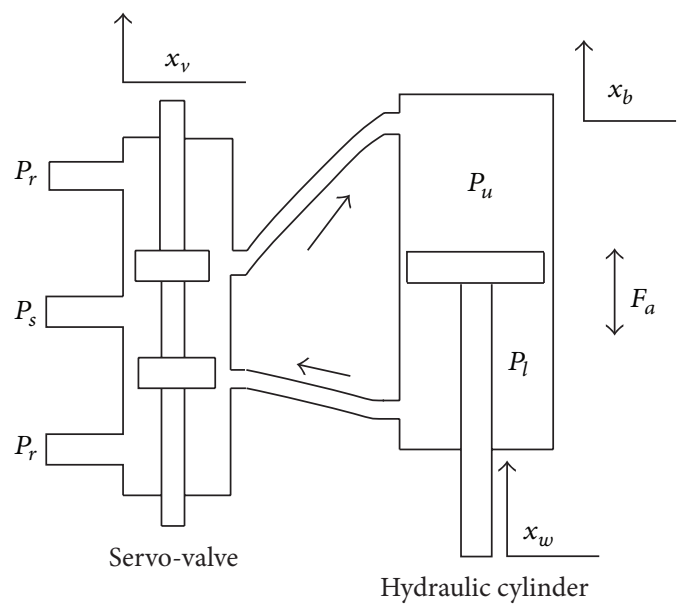

Figure 2: The electrohydraulic actuator system.

introduction of such a hydraulic actuator can be found in [23]. The spool valve system is formulated as

$$
\dot{x}_{v}=\frac{1}{\tau}\left(-x_{v}+K_{c} u\right),
$$

where $u$ is the control voltage and $\tau$ is the mechanical delay time constant of the servo-valve system. $K_{c}$ is the conversion gain.

The dynamic of the hydraulic cylinder equation is given by [24]

$$
\dot{F}_{a}=-\beta F_{a}-\alpha A^{2}\left(\dot{x}_{b}-\dot{x}_{w}\right)+\gamma A x_{v} \sqrt{P_{s}-\frac{F_{a} \operatorname{sgn}\left(x_{v}\right)}{A}},
$$

where $A$ is area of piston, meanwhile, $\alpha, \beta$, and $\gamma$ are the hydraulic parameters which may be time varying. Consider $\alpha=4 \beta_{e} / V_{t}, \beta=\alpha C_{t p}$, and $\gamma=\alpha C_{d} w \sqrt{1 / \rho}$, where $\beta_{e}$ is bulk modulus of hydraulic fluid, $V_{t}$ is the total volume of actuator cylinder chamber, $C_{t p}$ is the leakage coefficient, $C_{d}$ is the discharge coefficient, $w$ is the spool valve area gradient, 
$\rho$ is the hydraulic fluid density, and $P_{s}$ is the hydraulic supply pressure.

Remark 1 (see [25]). The practical hydraulic actuator has the properties of inherent nonlinear and time-varying characteristics, and precise parameters in (3) are not easy to estimate which is due to the uncertainties in the actuator dynamic model. Furthermore, the uncertainties in the model are bounded.

Select the state variables as $x=\left[x_{1}, x_{2}, x_{3}, x_{4}\right]^{T}$, where $x_{1}=x_{b}$, the camera (sprung mass) displacement; $x_{2}=\dot{x}_{b}$, camera (sprung mass) velocity; $x_{3}=x_{w}$, unsprung mass displacement; $x_{4}=\dot{x}_{w}$, unsprung mass velocity; $x_{r}$ is external disturbances; and $y=\left[x_{1}, x_{2}, x_{3}, x_{4}\right]^{T}$. Then, the equation of quarter-car active suspension system with actuator dynamic is given as

$$
\dot{x}=A x+B F_{a}+B_{w} x_{r}
$$

where

$$
\begin{aligned}
A & =\left[\begin{array}{cccc}
0 & 1 & 0 & 0 \\
-\frac{k_{s}}{m_{b}} & -\frac{c_{p}}{m_{b}} & \frac{k_{s}}{m_{b}} & \frac{c_{p}}{m_{b}} \\
0 & 0 & 0 & 1 \\
\frac{k_{s}}{m_{w}} & \frac{c_{p}}{m_{w}} & -\frac{k_{s}+k_{t}}{m_{w}} & -\frac{c_{p}}{m_{w}}
\end{array}\right], \\
B & =\left[\begin{array}{c}
0 \\
\frac{1}{m_{b}} \\
0 \\
-\frac{1}{m_{w}}
\end{array}\right], \\
B_{w} & =\left[\begin{array}{c}
0 \\
0 \\
0 \\
\frac{k_{t}}{m_{w}}
\end{array}\right] .
\end{aligned}
$$

Actually, the sprung mass is always time varying. The system dynamics can be simplified as

$$
\ddot{x}_{1}=f(x, t)+g(t) F_{a},
$$

where $f(x, t)$ is an unknown bounded function and $g(t)$ is the time-varying control gain. From (4), we can obtain

$$
\begin{aligned}
f(x, t)= & \left(-\frac{k_{s}}{m_{b}}\right) x_{1}-\left(\frac{c_{p}}{m_{b}}\right) x_{2}+\left(\frac{k_{s}}{m_{b}}\right) x_{3} \\
& +\left(\frac{c_{p}}{m_{b}}\right) x_{4}, \\
g(t)= & \frac{1}{m_{b}} .
\end{aligned}
$$

The objective of this paper is to design a controller to suppressing vertical vibration of the camera so that the target is stable in image plane. Since the camera is in rigid connection with the car body, the displacement of the car body $x_{1}$ can reflect the vibration situation of the camera in the following analysis.

Lemma 2 (see [26]). Let $f(x)$ be a continuous function, which is defined on a compact set $D$. Then, there exists a neural networks system $W^{T} z(x)$ which can approximate $f(x)$ with arbitrary accuracy, such that

$$
f(x)=W^{T} z(x)+\varepsilon(x),
$$

where $W=\left[w_{1}, w_{2}, \ldots, w_{N}\right]^{T}$ is the ideal constant weight vector, $z(x)=\left[z_{1}(x), z_{2}(x), \ldots, z_{N}(x)\right]^{T}$ is the RBFs vector, $N>1$ is the number of the neurons, and $\varepsilon(x)$ is the approximation error which is minimized by the ideal vector $W$ :

$$
W:=\arg \min _{W \in \mathbb{R}}\left\{\sup _{x \in D}\left|f(x)-W^{T} z(x)\right|\right\} .
$$

Assuming that $\varepsilon(x)$ is bounded, $|\varepsilon(x)|<\varepsilon^{*}<\infty$ with $\varepsilon^{*}$ being an unknown constant. $z_{i}(x)$ is Gaussian function; that is,

$$
z_{i}(x)=\exp \left[\frac{-\left(x-\mu_{i}\right)^{T}\left(x-\mu_{i}\right)}{\eta_{i}^{2}}\right]
$$

with $\mu=\left[\mu_{1}, \mu_{2}, \ldots, \mu_{N}\right]^{T}$ and $\eta_{i}$ representing the centers and widths of the Gaussian functions, and $n$ is the dimension of $x$.

\section{Controller Design}

The proposed control scheme consists of two loops as shown in Figure 3. The nonlinearity of the hydraulic actuators and their force generation capabilities are also considered in control design. The outer loop performs a position control of the vehicle body, and the desired value of $x_{d}$ is zero. The inner loop is the PID controller for tracking purpose. The RBFNN approach is employed to approximate the unknown function $f(x, t)$ and $g(t)$. This control method may be much more feasible in practical application.

3.1. Designing Inner Loop. In order to track the desired force for the inner loop, a PID controller is used to control the electrohydraulic actuators. The PID controller can be designed to stabilize the closed loop system and provide suitable tracking performance [27]. The PID controller is given as follows:

$$
u=k_{p} e_{F}+k_{i} \int_{0}^{t} e_{F} d t+k_{d} \frac{d e_{F}}{d t},
$$

where $k_{p}$ is the proportional gain, $k_{i}$ is the integral gain, and $k_{d}$ is the differential gain. The error is defined as

$$
e_{F}=F_{d}-F_{a}
$$

where $F_{d}$ is the desired force and $F_{a}$ is the actual force generated by the actuator. 


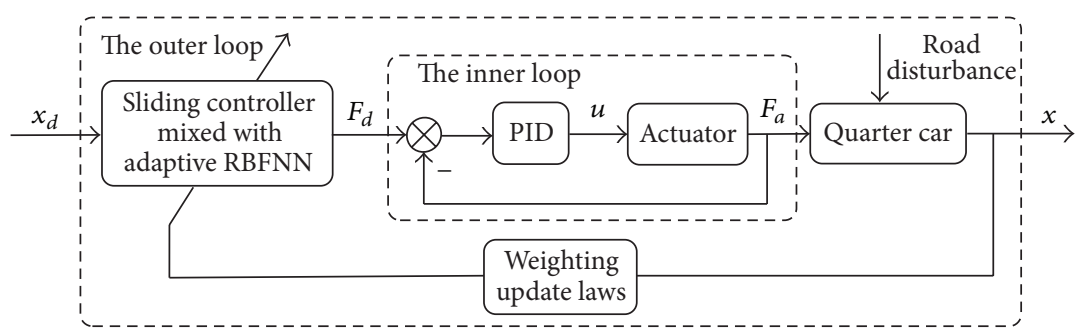

FIgURE 3: The system control block diagram.

3.2. Designing Outer Loop. According to RFB neural network stated previously, a novel sliding mode controller for a quarter-car active suspension systems can be developed. The sliding surface is defined as

$$
s=\dot{x}_{1}+\lambda x_{1},
$$

where $\lambda$ is the convergent rate of $x_{1}$ on the sliding surface. The derivative of above equation (13) is given as follows:

$$
\dot{s}=\ddot{x}_{1}+\lambda \dot{x}_{1} .
$$

Substituting (6) into (14),

$$
\dot{s}=f(x, t)+g(t) F_{d}+\lambda \dot{x}_{1} .
$$

The control force $F_{d}$ can be designed as

$$
F_{d}=\frac{1}{\widehat{g}}\left(-\widehat{f}-\hat{\lambda} \dot{x}_{1}-\eta_{1} s-\eta_{2} \operatorname{sgn}(s)\right),
$$

where $\hat{\lambda}, \hat{f}$, and $\hat{g}$ are the estimate of $\lambda, f(x, t)$, and $g(t)$, respectively. The constants $\eta_{1}>0$ and $\eta_{2}>0$ are parameters to be selected. Substituting (16) into (15),

$$
\begin{aligned}
\dot{s}= & -\eta_{1} s-\eta_{2} \operatorname{sgn}(s)+(f-\widehat{f})+(g-\widehat{g}) F_{d} \\
& +(\lambda-\widehat{\lambda}) \dot{x}_{1}, \\
\dot{s}= & -\eta_{1} s-\eta_{2} \operatorname{sgn}(s)+\widetilde{f}+\widetilde{g} F_{d}+\tilde{\lambda} \dot{x}_{1},
\end{aligned}
$$

where $\tilde{f}=f-\widehat{f}, \widetilde{g}=g-\widehat{g}$, and $\tilde{\lambda}=\lambda-\widehat{\lambda}$. They are assumed to be unknown bounded function and satisfy the Dirichlet conditions. Therefore, the RBFNN can represent $\tilde{f}$ and $\tilde{g}$ as

$$
\begin{aligned}
& \tilde{f}=\widetilde{W}_{f}^{T} z_{f}(x)+\varepsilon_{f}, \\
& \tilde{g}=\widetilde{W}_{g}^{T} z_{g}(x)+\varepsilon_{g},
\end{aligned}
$$

where $\widetilde{W}_{f}^{T}=W_{f}^{T}-\widehat{W}_{f}^{T}$ and $\widetilde{W}_{g}^{T}=W_{g}^{T}-\widehat{W}_{g}^{T}$. With these representations, (18) can be rewritten as

$$
\begin{aligned}
\dot{s}= & -\eta_{1} s-\eta_{2} \operatorname{sgn}(s)+\widetilde{W}_{f}^{T} z_{f}(x)+\widetilde{W}_{g}^{T} z_{g}(x) F_{d} \\
& +\widetilde{\lambda}_{\dot{x}_{1}}+\varepsilon_{a},
\end{aligned}
$$

where $\varepsilon_{a}=\varepsilon_{f}+F_{d} \varepsilon_{g}$. To prove the stability of this control system and to find the update laws for $\dot{\widehat{W}}_{f}$ and $\dot{\widehat{W}}_{g}$, the Lyapunov function candidate is chosen as

$$
V=\frac{1}{2} s^{2}+\frac{1}{2} \widetilde{\lambda}^{2}+\frac{1}{2} \widetilde{W}_{f}^{T} Q_{f} \widetilde{W}_{f}+\frac{1}{2} \widetilde{W}_{g}^{T} Q_{g} \widetilde{W}_{g}
$$

where $Q_{f}$ and $Q_{g}$ are both positive definite and symmetric matrix. Taking the time derivative of (21) yields

$$
\begin{aligned}
\dot{V}= & s \dot{s}+\tilde{\lambda} \dot{\widetilde{\lambda}}-\widetilde{W}_{f}^{T} Q_{f} \dot{\widehat{W}}_{f}-\widetilde{W}_{g}^{T} Q_{g} \dot{\widehat{W}}_{g}, \\
\dot{V}= & -\eta_{1} s^{2}-\eta_{2}|s|+s \varepsilon_{a}+\widetilde{W}_{f}^{T}\left(s z_{f}-Q_{f} \dot{\widehat{W}}_{f}\right) \\
& +\widetilde{W}_{g}^{T}\left(s z_{g} F_{d}-Q_{g} \dot{\widehat{W}}_{g}\right)+\tilde{\lambda}\left(s \dot{x}_{1}+\dot{\hat{\lambda}}\right) .
\end{aligned}
$$

Therefore, the update laws and parameter $\dot{\hat{\lambda}}$ can be designed as

$$
\begin{aligned}
\dot{\widehat{W}}_{f} & =Q_{f}^{-1} s z_{f}, \\
\dot{\widehat{W}}_{g} & =Q_{g}^{-1} s z_{g} F_{d}, \\
\dot{\hat{\lambda}} & =-s \dot{x}_{1} .
\end{aligned}
$$

So $\widehat{f}, \widehat{g}$, and $\hat{\lambda}$ could be approximated by the following equations:

$$
\begin{aligned}
& \widehat{f}=\widehat{W}_{f}^{T} z_{f}(x), \\
& \widehat{g}=\widehat{W}_{g}^{T} z_{g}(x), \\
& \widehat{\lambda}=\int-s \dot{x}_{1} d t .
\end{aligned}
$$

Hence, we may conclude that

$$
\dot{V}=-\eta_{1} s^{2}-\eta_{2}|s|+s \varepsilon_{a} .
$$

In order to satisfy $\dot{V} \leq 0$, it must be $\eta_{2}|s| \geq s \varepsilon_{a}$.

Then, we can choose $\eta_{2}$ with the condition $\eta_{2} \geq\left|\varepsilon_{a}\right|$. The derivative of the Lyapunov function becomes

$$
\dot{V} \leq-\eta_{1} s^{2} \leq 0
$$

Therefore, $s, \widetilde{W}_{f}, \widetilde{W}_{g} \in L_{\infty}$, and $\int_{0}^{\infty} s^{2} d t \leq$ $-\eta^{-1} \int_{0}^{\infty} \dot{V} d t<\infty$ imply $s \in L_{2}$. In addition, (20) implies $\dot{s} \in L_{\infty}$; hence, asymptotic convergence of $s$ can be concluded using Barbalat's lemma [28]. And this further implies that the sliding surface can converge to zero at last. 
TABLE 1: Parameter values of the active suspension system.

\begin{tabular}{lc}
\hline Parameter & Value \\
\hline$m_{b}$ & $290 \mathrm{~kg}$ \\
$m_{w}$ & $59 \mathrm{~kg}$ \\
$k_{s}$ & $16812 \mathrm{~N} / \mathrm{m}$ \\
$k_{t}$ & $190000 \mathrm{~N} / \mathrm{m}$ \\
$c_{p}$ & $1000 \mathrm{~N} / \mathrm{m} / \mathrm{s}$ \\
$A$ & $3.35 \times 10^{-4} \mathrm{~m}^{2}$ \\
$\alpha$ & $4.515 \times 10^{13} \mathrm{~N} / \mathrm{m}^{5}$ \\
$\beta$ & $1 \mathrm{~s}^{-1}$ \\
$\gamma$ & $1.545 \times 10^{9} \mathrm{~N} / \mathrm{m}^{5 / 2} \mathrm{~kg}^{1 / 2}$ \\
$P_{s}$ & $10342500 \mathrm{~Pa}$ \\
$T$ & $0.003 \mathrm{~s}$ \\
$K_{c}$ & $0.001 \mathrm{~m} / \mathrm{V}$ \\
\hline
\end{tabular}

\section{Simulations}

In this section, the simulation will be given on a quarter-car model. System parameters are shown in Table 1.

In order to evaluate the performance of the designed controller, we consider three typical cases.

Case 1. As the resonance frequency of the car body is about $1 \mathrm{~Hz}$, an external excitation close to this frequency might induce unwanted oscillation. Then, to test control performance near the system resonance frequency, consider that the road profile is assigned as

$$
x_{r 1}(t)=0.025 \sin 2 \pi t .
$$

Case 2. Consider a sine bump in an otherwise smooth road surface. The mathematical description of this type ground displacement is given by

$$
x_{r 2}(t)= \begin{cases}0.5 h\left(1+\sin \left(\frac{2 \pi v}{L} t\right)\right) & \text { if } t_{l} \leq t \leq t_{h} \\ 0 & \text { otherwise }\end{cases}
$$

where $h$ and $L$ are height and length of the bump and $v$ is the vehicle velocity. Assume $h=0.05 \mathrm{~m}, L=2.5 \mathrm{~m}$, and $v=18 \mathrm{~km} / \mathrm{h}$. The corresponding road excitation is shown in Figure 4.

Case 3. Consider the road excitation which is consistent and typically specified as a random process with a ground displacement power spectral density (PSD) of

$$
\begin{aligned}
G_{q}(n) & =G_{q}\left(n_{0}\right)\left(\frac{n}{n_{0}}\right)^{-W}, \\
G_{q}(f) & =\frac{1}{v} G_{q}(n),
\end{aligned}
$$

where $n$ is the spatial frequency in $m^{-1}, G_{q}(f)$ stands for PSD in time domain, and $n_{0}$ is the reference spatial frequency. Select the road roughness as $G_{q}\left(n_{0}\right)=64 \times 10^{-6} \mathrm{~m}^{3}, n_{0}=0.1$, $W=2$, and $v=20 \mathrm{~km} / \mathrm{h}$. This PSD indicates that the road profile can be obtained from integrating a white noise in time

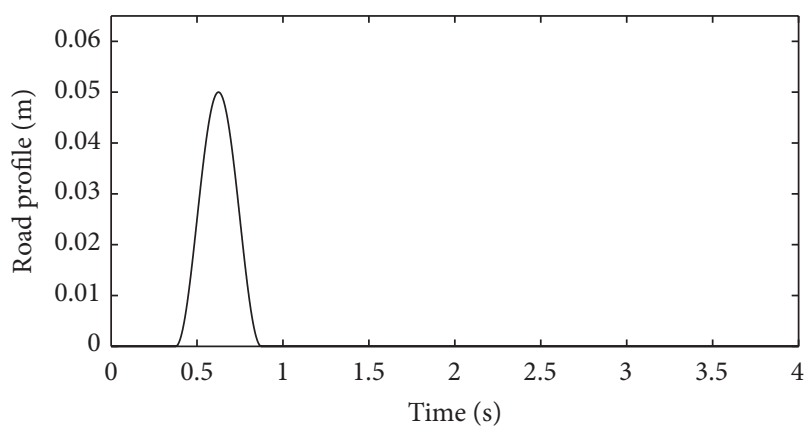

Figure 4: Bump road profile.

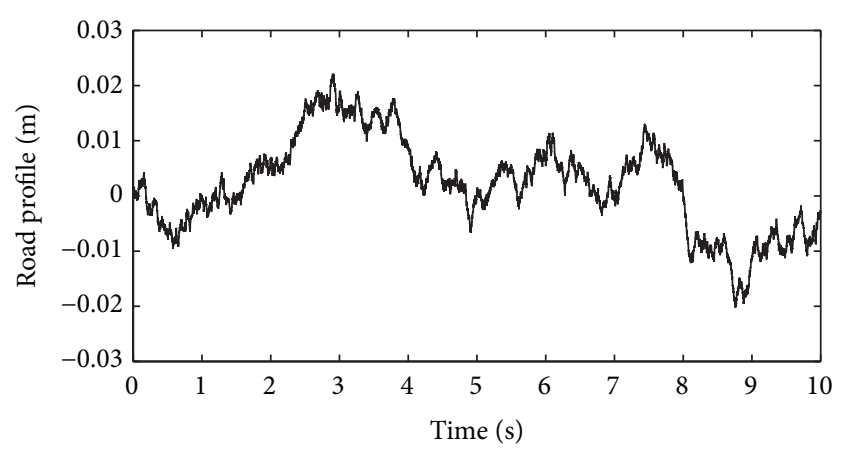

FIGURE 5: Random road profile.

domain [29]. So the displacement of the road excitation in time domain is shown in Figure 5.

In the simulation, the method presented in this paper is represented by NN-SMC, and the compared method is denoted by LQR. The matrixes $Q_{f}$ and $Q_{g}$ are chosen as diagonal matrixes and all of these diagonal elements are 0.3 and 0.2 , respectively. The initial parameter $\hat{\lambda}$ that influences the converging slope of the sliding surface is chosen as 100 . It will influence the converging rate of $x_{1}$ on the sliding surface. We set the adaptation laws with initial conditions $\eta_{1}=1$ and $\eta_{2}=1 \times 10^{4}$. The initial weighting vectors are selected to be $\widehat{W}_{f}^{T}(0)=\left[\begin{array}{lllll}0.5 & 0.5 & 0.5 & 0.5 & 0.5\end{array}\right]^{T}$ and $\widehat{W}_{g}^{T}(0)=\left[\begin{array}{lllll}0.5 & 0.5 & 0.5 & 0.5 & 0.5\end{array}\right]^{T}$, respectively, after a rough test, and the system initial condition is set as $x(0)=$ $\left[\begin{array}{llll}0.1 & 0.1 & 0.1 & 0.1\end{array}\right]^{T}$. The PID controllers for all the three cases are $k_{p}=10^{-4}, k_{i}=10^{-7}$, and $k_{d}=10^{-7}$. The number of neurons in the hidden layer is 5 , and $z_{f}(x)=z_{g}(x)=$ $\left[z_{1}(x), z_{2}(x), \ldots, z_{5}(x)\right]^{T}$. The center of Gaussian function is set $\delta_{i}=5,(i=1,2, \ldots 5)$, and $\mu_{1}=-1, \mu_{2}=-0.5, \mu_{3}=0$, $\mu_{4}=0.5$, and $\mu_{5}=1$.

According to the numerical result of sinusoidal road excitation in Figure 6, the displacement attenuation of the camera (sprung mass) with the proposed NN-SMC controller is dramatically improved and the maximum deflection is kept within $\pm 0.2 \mathrm{~mm}$. It can be observed that the maximum camera displacement has been reduced from $60 \mathrm{~mm}$ to $0.1 \mathrm{~mm}$. And the suspension deflection is almost the same 


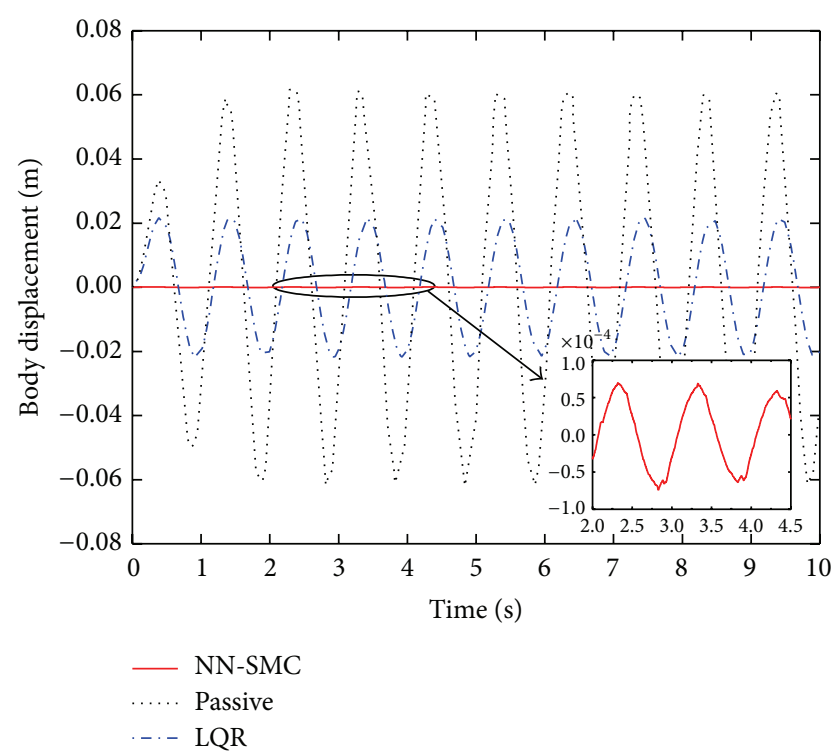

Figure 6: Body displacement (Case 1).

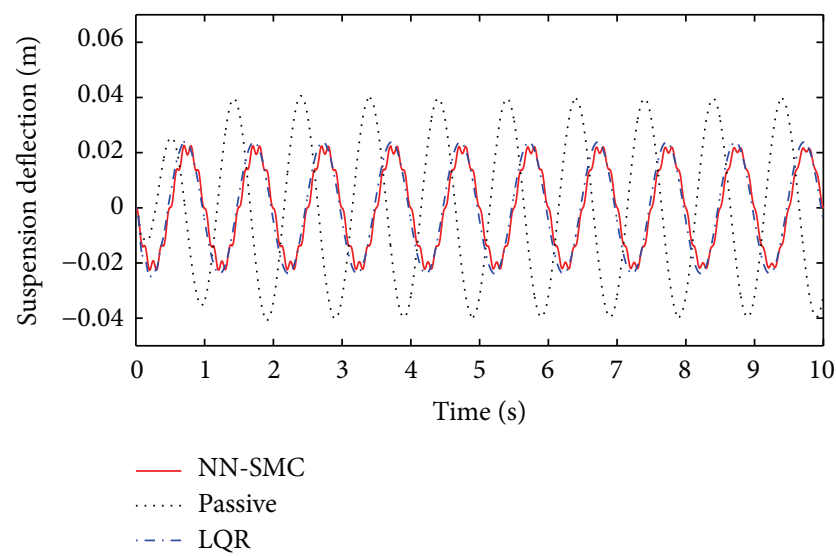

FIgURE 7: Suspension deflection (Case 1).

as that with the LQR method in Figure 7, but the maximum magnitude is about $50 \%$ smaller than the passive suspension. From Figure 8, the estimated value of $f(x)$ converges fast to true value of $f(x)$ within $0.6 \mathrm{~s}$.

When a vehicle is riding on the bump terrain, the dynamic responses of the camera position by using NNSMC and LQR controllers are shown in Figure 9. It can be observed that NN-SMC has more obvious performance improvement. The maximum amplitude of sprung mass displacement responses is suppressed by $65 \%$ and $30 \%$, respectively, compared with the passive suspension, and the curve converges to zero within $1.6 \mathrm{~s}$ by NN-SMC method. In Figure 10, the maximum value of suspension deflection by $\mathrm{NN}-\mathrm{SMC}$ is $35 \mathrm{~mm}$, which is smaller than the value under LQR method. In addition, the trajectory of $\hat{\lambda}$ is shown in Figure 11.

The dynamic responses of the sprung mass displacement with the random excitation are shown in Figure 12. The root mean square (RMS) value of the sprung mass displacement

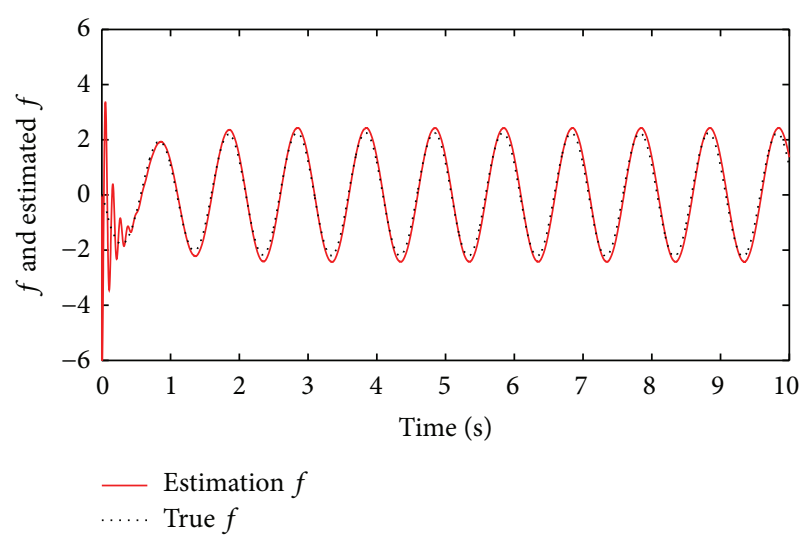

Figure 8: True and estimation $f(x)$ (Case 1).

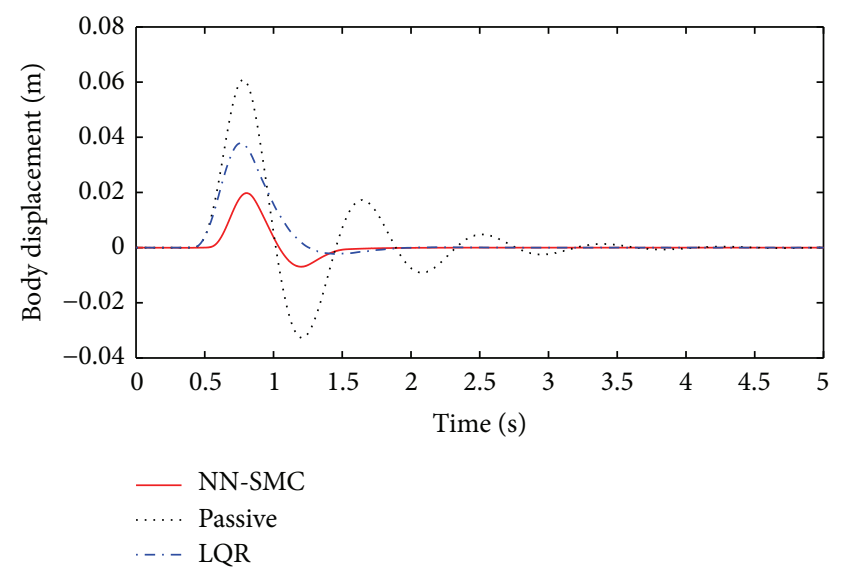

FIgure 9: Body displacement (Case 2).

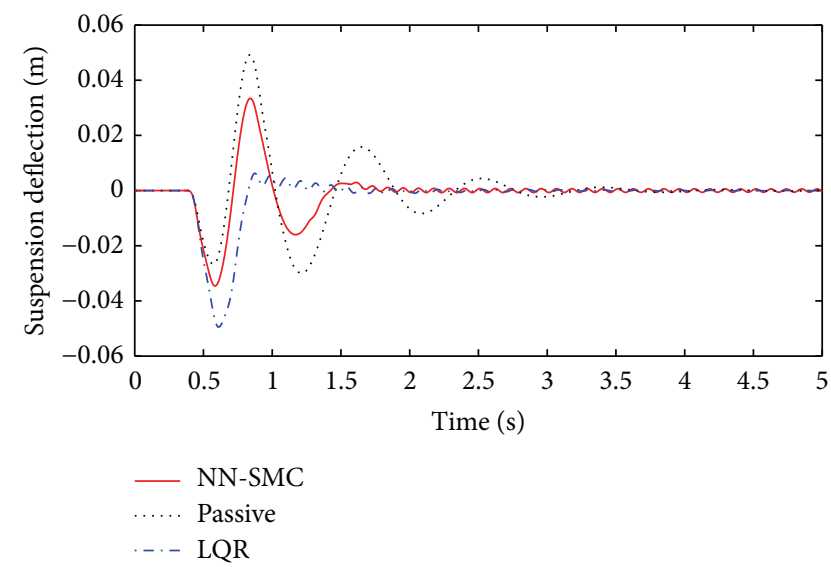

FIGURE 10: Suspension deflection (Case 2).

with NN-SMC is $3.33 \times 10^{-4}$, while the RMS value is $8.7 \times 10^{-3}$ by LQR. As it appears in Figure 13, the fluctuation amplitude of the red line with NN-SMC is smaller than the blue dotted line. In Figure 14, the maximum value of body acceleration by NN-SMC is $0.65 \mathrm{~m} / \mathrm{s}^{2}$, which is smaller than the maximum value under LQR method. But the root mean square (RMS) 


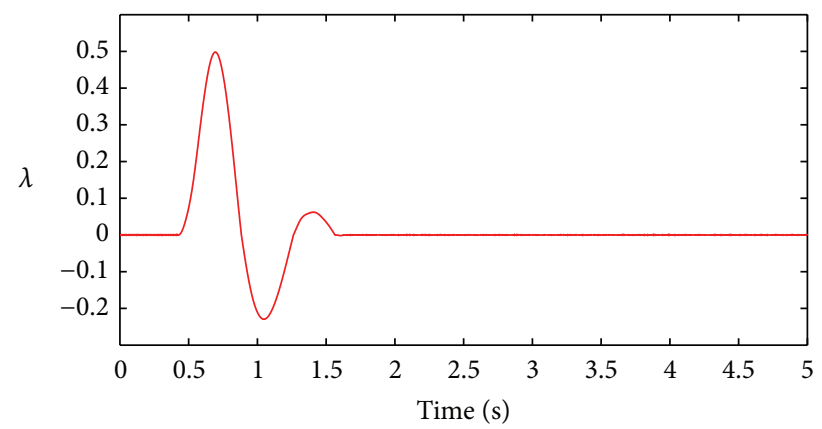

FIgURE 11: Estimate of $\lambda$ (Case 2).

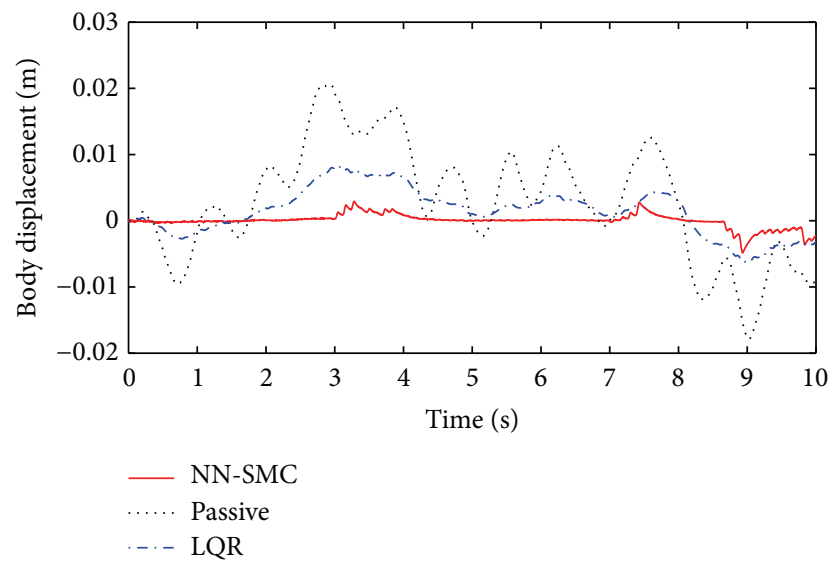

Figure 12: Body displacement (Case 3).

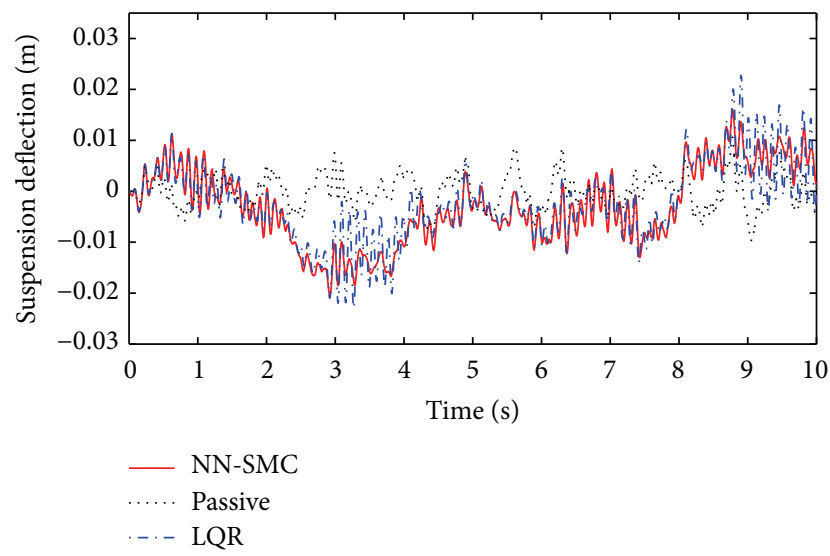

Figure 13: Suspension deflection (Case 3).

value of the body acceleration with NN-SMC is $4.1 \times 10^{-2}$, which is slightly larger than the RMS value $3.7 \times 10^{-2}$ by LQR.

From the above results of the simulation, it is clearly seen from Figures 9 and 10 in Case 2 that the closed loop system with NN-SMC has stronger robustness. Furthermore, Figure 6 indicates that the vertical vibration of the camera is much smaller in the NN-SMC method for the resonance frequency of the car body, which improves the stabilization for capturing images. The tracking speed of the estimation value of $f(x)$ is given in Figure 8 and the tracking performance

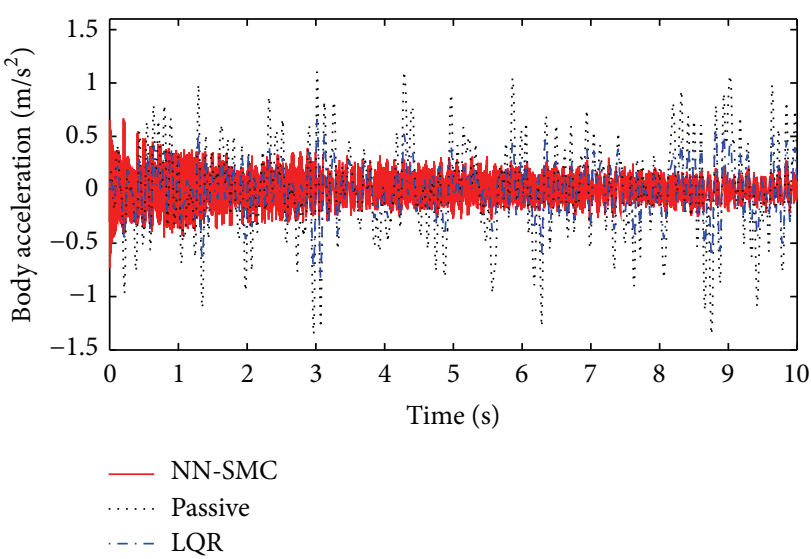

Figure 14: Body acceleration (Case 3).

is satisfactory. Therefore, the NN-SMC method possesses a faster dynamic property and has a better performance on the whole.

\section{Conclusions}

The vibration of the camera will directly affect the image quality and impede the subsequent processes. So the study of the image vibration attenuation is important in applications. In this paper, this innovative sliding mode control based on mixed adaptive neural networks approach has been successfully employed and simulated to reduce the vertical vibration of the camera using a quarter-car active suspension system, where electrohydraulic actuators with nonlinear characteristics are considered for accurate control. The radial basis function (RBF) network is used to overcome the uncertainties in the dynamic model of ASS, and, by means of the Lyapunov theorem, the weights of neural networks are regulated online without previous learning phase. The whole control architecture contains two loops. The inner loop is a PID controller to track the desired force, whereas, in the outer loop, the position controller is designed by the sliding mode strategy. The proposed control scheme can significantly suppress the displacement oscillation amplitude of the camera. Different road conditions are used in the simulation. The results show that the presented method can guarantee that the active suspension system has a fast convergence and strong robustness, which can effectively enhance the stabilization of the camera image.

\section{Conflict of Interests}

The authors declare that there is no conflict of interests regarding the publication of this paper.

\section{Acknowledgments}

This research is partially supported by the National Science Foundation of China under Contract no. 51005018, and this paper is also funded by International Graduate Exchange Program of Beijing Institute of Technology. 


\section{References}

[1] J. S. Jin, Z. Zhu, and G. Xu, "A stable vision system for moving vehicles," IEEE Transactions on Intelligent Transportation Systems, vol. 1, no. 1, pp. 32-39, 2000.

[2] P. Rawat and J. Singhai, "Review of motion estimation and video stabilization techniques for hand held mobile video," Signal \& Image Processing, vol. 2, no. 2, p. 159, 2011.

[3] S. Ertürk, "Real-time digital image stabilization using Kalman filters," Real-Time Imaging, vol. 8, no. 4, pp. 317-328, 2002.

[4] Y.-C. Chang and J. Shaw, "Low-frequency vibration control of a pan/tilt platform with vision feedback," Journal of Sound and Vibration, vol. 302, no. 4-5, pp. 716-727, 2007.

[5] D. Cao, X. Song, and M. Ahmadian, "Editors' perspectives: road vehicle suspension design, dynamics, and control," Vehicle System Dynamics, vol. 49, no. 1-2, pp. 3-28, 2011.

[6] D. Hrovat, "Survey of advanced suspension developments and related optimal control applications," Automatica, vol. 33, no. 10, pp. 1781-1817, 1997.

[7] S. Ryu, Y. Park, and M. Suh, "Ride quality analysis of a tracked vehicle suspension with a preview control," Journal of Terramechanics, vol. 48, no. 6, pp. 409-417, 2011.

[8] W. Sun, H. Gao, and B. Yao, "Adaptive robust vibration control of full-car active suspensions with electrohydraulic actuators," IEEE Transactions on Control Systems Technology, vol. 21, no. 6, pp. 2417-2422, 2013.

[9] H. Gao, W. Sun, and P. Shi, "Robust sampled-data $H_{\infty}$ control for vehicle active suspension systems," IEEE Transactions on Control Systems Technology, vol. 18, no. 1, pp. 238-245, 2010.

[10] G. Verros, S. Natsiavas, and C. Papadimitriou, "Design optimization of quarter-car models with passive and semi-active suspensions under random road excitation," Journal of Vibration and Control, vol. 11, no. 5, pp. 581-606, 2005.

[11] X. Shen and H. Peng, "Analysis of active suspension systems with hydraulic actuators," in The Dynamics of Vehicles on Roads and on Tracks Supplement to Vehicle System Dynamics: Proceedings of the 18th Iavsd Symposium Held in Kanagawa, Japan August 24-30, 2003, vol. 41, CRC Press, 2005.

[12] H. Chen, Z.-Y. Liu, and P.-Y. Sun, "Application of constrained script $H_{\infty}$ control to active suspension systems on half-car models," Journal of Dynamic Systems, Measurement and Control, vol. 127, no. 3, pp. 345-354, 2005.

[13] M. P. Nagarkar, G. J. Vikhe, K. R. Borole, and V. M. Nandedkar, "Active control of quarter-car suspension system using linear quadratic regulator," International Journal of Automotive and Mechanical Engineering, vol. 3, no. 1, pp. 364-372, 2011.

[14] H. Li, H. Liu, H. Gao, and P. Shi, "Reliable fuzzy control for active suspension systems with actuator delay and fault," IEEE Transactions on Fuzzy Systems, vol. 20, no. 2, pp. 342-357, 2012.

[15] Ş. Yildirim and I. Uzmay, "Neural network applications to vehicle's vibration analysis," Mechanism and Machine Theory, vol. 38, no. 1, pp. 27-41, 2003.

[16] U. N. L. T. Alves, J. P. F. Garcia, M. C. M. Teixeira, S. C. Garcia, and F. B. Rodrigues, "Sliding mode control for active suspension system with data acquisition delay," Mathematical Problems in Engineering, vol. 2014, Article ID 529293, 13 pages, 2014.

[17] R.-J. Lian, "Enhanced adaptive self-organizing fuzzy slidingmode controller for active suspension systems," IEEE Transactions on Industrial Electronics, vol. 60, no. 3, pp. 958-968, 2013.

[18] A.-C. Huang and Y.-S. Kuo, "Sliding control of non-linear systems containing time-varying uncertainties with unknown bounds," International Journal of Control, vol. 74, no. 3, pp. 252264, 2001.

[19] Y. Jin and D. J. Yu, "Adaptive neuron control using an integrated error approach with application to active suspensions," International Journal of Automotive Technology, vol. 9, no. 3, pp. 329335, 2008.

[20] M. Chen, S. S. Ge, and B. V. E. How, "Robust adaptive neural network control for a class of uncertain MIMO nonlinear systems with input nonlinearities," IEEE Transactions on Neural Networks, vol. 21, no. 5, pp. 796-812, 2010.

[21] S. S. Ge and C. Wang, "Adaptive neural control of uncertain MIMO nonlinear systems," IEEE Transactions on Neural Networks, vol. 15, no. 3, pp. 674-692, 2004.

[22] M. M. Fateh and M. M. Zirkohi, "Adaptive impedance control of a hydraulic suspension system using particle swarm optimisation," Vehicle System Dynamics, vol. 49, no. 12, pp. 1951-1965, 2011.

[23] M.-M. Ma and H. Chen, "Disturbance attenuation control of active suspension with non-linear actuator dynamics," IET Control Theory and Applications, vol. 5, no. 1, pp. 112-122, 2011.

[24] A. G. Alleyne and R. Liu, "Systematic control of a class of nonlinear systems with application to electrohydraulic cylinder pressure control," IEEE Transactions on Control Systems Technology, vol. 8, no. 4, pp. 623-634, 2000.

[25] P.-C. Chen and A.-C. Huang, "Adaptive sliding control of active suspension systems with uncertain hydraulic actuator dynamics," Vehicle System Dynamics, vol. 44, no. 5, pp. 357-368, 2006.

[26] M. M. Polycarpou, "Stable adaptive neural control scheme for nonlinear systems," IEEE Transactions on Automatic Control, vol. 41, no. 3, pp. 447-451, 1996.

[27] M. H. Ab Talib and I. Z. Mat Darns, "Self-tuning PID controller for active suspension system with hydraulic actuator," in Proceedings of the IEEE Symposium on Computers and Informatics (ISCI '13), pp. 86-91, April 2013.

[28] J.-J. E. Slotine and W. Li, Applied Nonlinear Control, vol. 199, Prentice-Hall, Englewood Cliffs, NJ, USA, 1991.

[29] F. Tyan, Y. F. Hong, S. H. Tu et al., "Generation of random road profiles," Journal of Advanced Engineering, vol. 4, no. 2, pp. 13731378, 2009. 

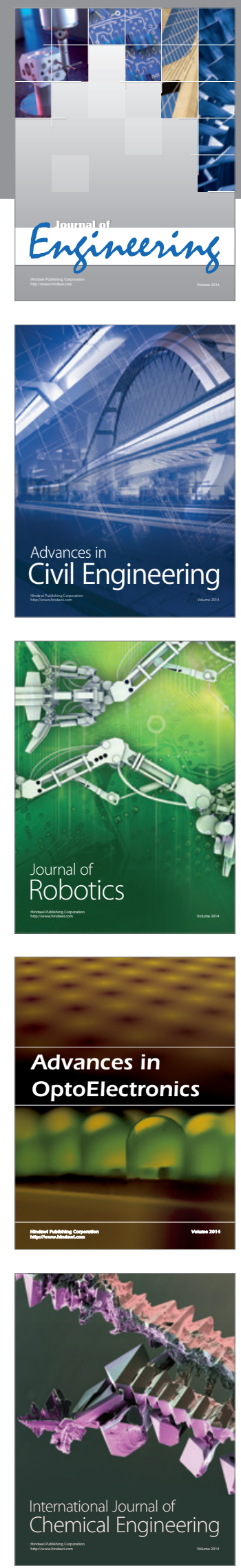

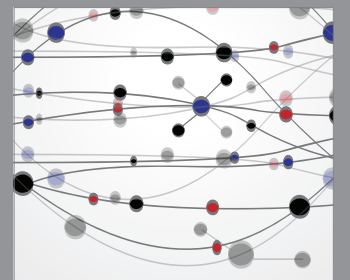

The Scientific World Journal
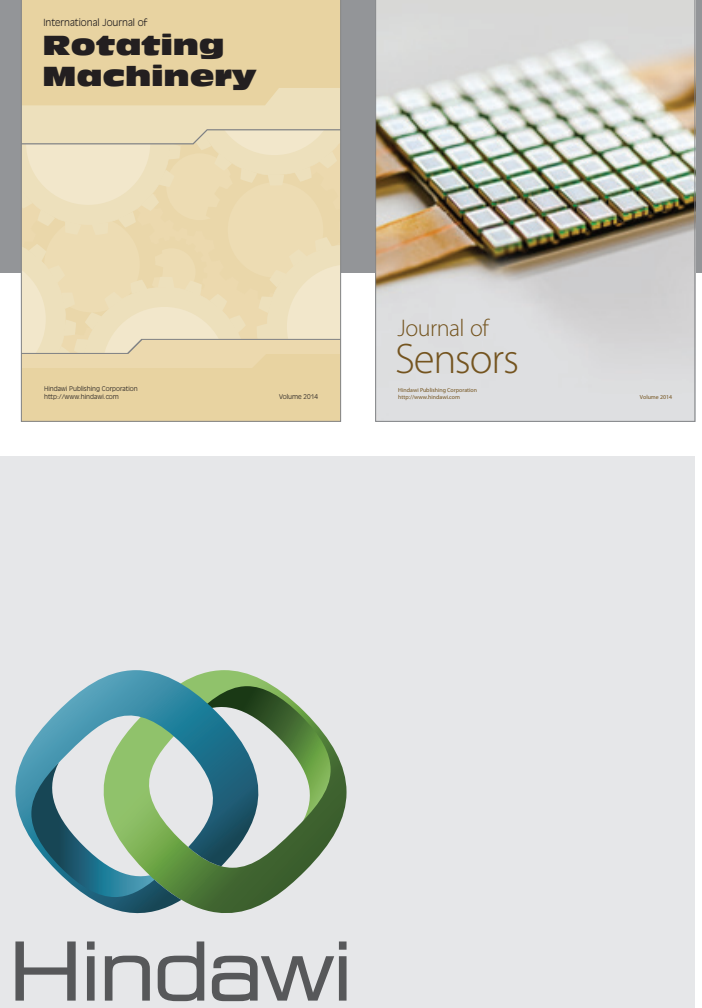

Submit your manuscripts at http://www.hindawi.com
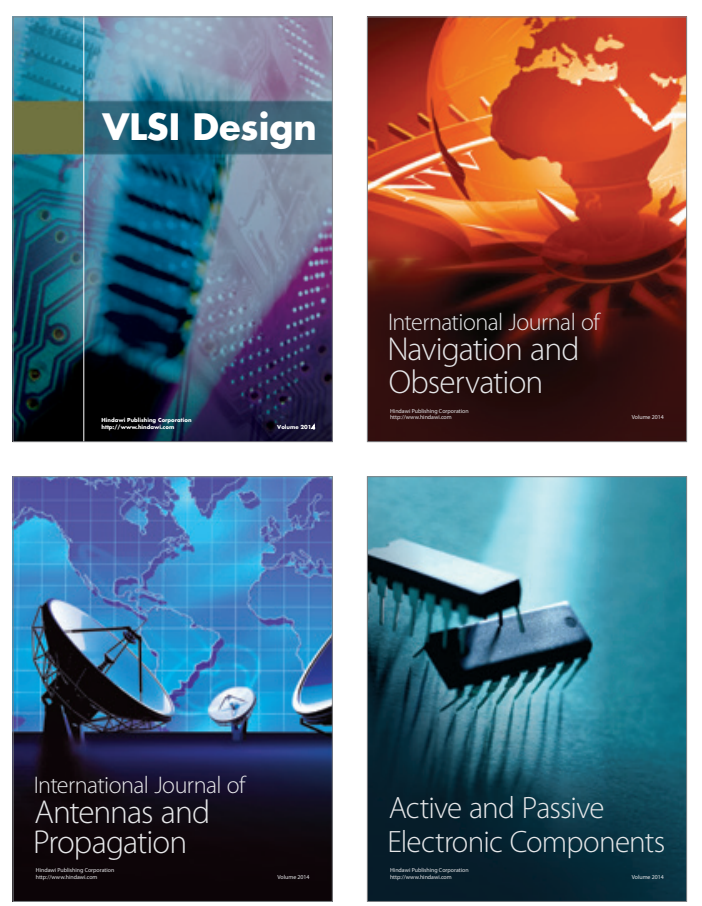
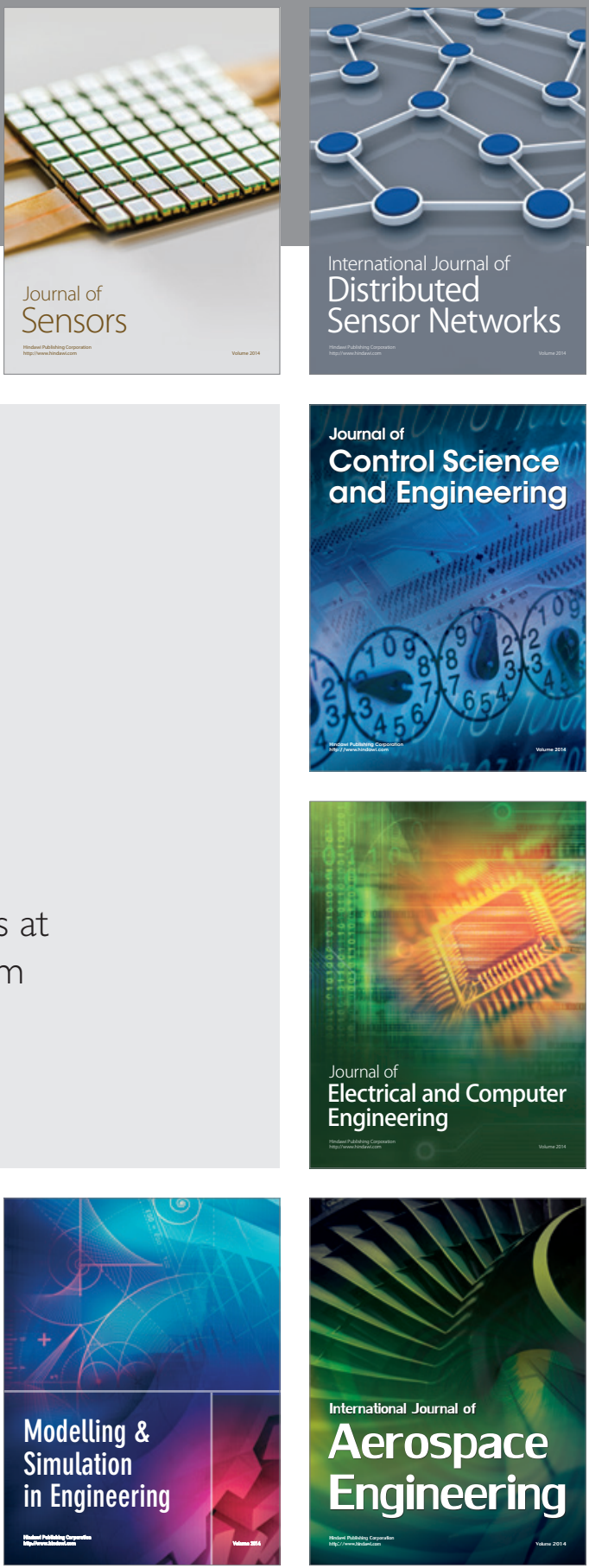

Journal of

Control Science

and Engineering
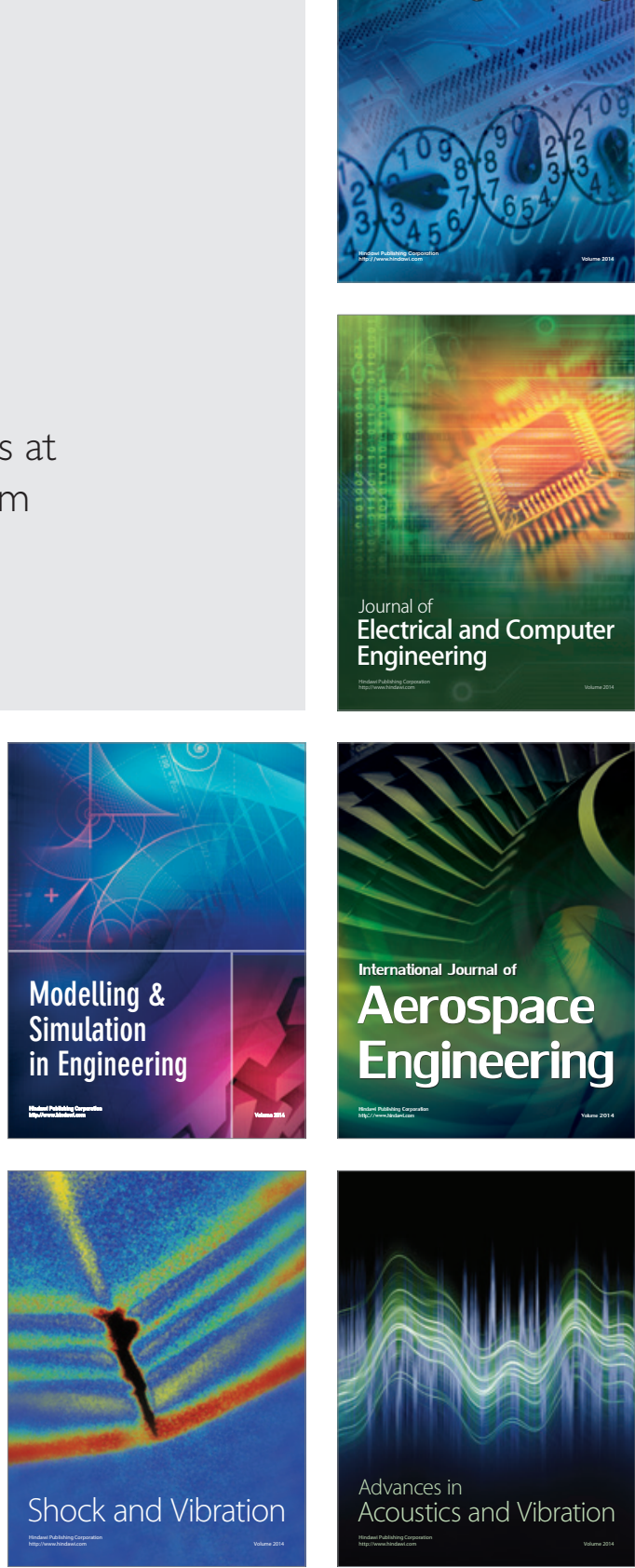